\title{
AMMC: Advanced Multi-core Memory Controller
}

\author{
Tassadaq Hussain ${ }^{1,2}$, Oscar Palomar ${ }^{1,2}$, Osman Unsal ${ }^{1}$, Adrian Cristal ${ }^{1,2,3}$, Eduard Ayguadé ${ }^{1,2}$, Mateo Valero ${ }^{1,2}$ \\ ${ }^{1}$ Computer Sciences, Barcelona Supercomputing Center, Barcelona, Spain \\ 2 Departament d'Arquitectura de Computadors, Universitat Politècnica de Catalunya, Barcelona, Spain \\ ${ }^{3}$ Artificial Intelligence Research Institute (IIIA), Centro Superior de Investigaciones Científicas (CSIC), Barcelona, Spain \\ Email: $\{$ first $\} .\{$ last $\} @$ bsc.es
}

\begin{abstract}
In this work, we propose an efficient scheduler and intelligent memory manager known as AMMC (Advanced Multi-Core Memory Controller), which proficiently handles data movement and computational tasks. The proposed AMMC system improves performance by managing complex data transfers at run-time and scheduling multi-cores without the intervention of a control processor nor an operating system. AMMC has been coupled with a heterogeneous system that provides both generalpurpose cores and application specific accelerators. The AMMC system is implemented and tested on a Xilinx ML505 evaluation FPGA board. The performance of the system is compared with a microprocessor based system that has been integrated with the Xilkernel operating system. Results show that the AMMC based multi-core system consumes $\mathbf{4 8 \%}$ less hardware resources, $\mathbf{2 7 . 9 \%}$ less on-chip power and achieves 6.8x of speed-up compared to the MicroBlaze-based multi-core system.
\end{abstract}

\section{INTRODUCTION}

Latest multi-core architectures require both programmability and performance and combine different types of cores, becoming heterogeneous systems. To get programmability, a part of the program is executed on general-purpose cores. To achieve performance and to increase power efficiency, compute intensive tasks are mapped into separate hardware accelerators or application-specific processors. The dedicated application specific accelerator cores have small footprint and low power dissipation and feature high performance [1].

To overcome the memory wall and to reduce the system power, a memory system is needed that supports cores with low frequency and low complexity, has efficient local memory and data management, with an intelligent scheduler while supporting a programming model that manages memory accesses in software so that hardware can best utilize them. In this work, we have integrated a memory controller with a heterogeneous multi-core system having Application Specific Hardware Accelerators (ASHA) and Scalar Soft Processor $(S S P)$, that we term AMMC (Advanced Multi-core Memory Controller). Some salient features of the AMMC are given below:

- The AMMC based system handles heterogeneous (SSP and ASHA) cores using Symmetric and Asymmetric scheduling policies, without the support of a master core nor operating system.

- Regular and irregular access patterns of heterogeneous multi-cores are described using a separate Descriptor Memory, which reduces the on-chip communication time and run-time address generation overhead.

- $\quad$ The AMMC Address Manager and Scheduler handles regular and irregular pattern requests of a heterogeneous multi-core system, provides precise timing and allows scheduling mode to be changed at runtime.

The research leading to these results has received funding from the European Research Council under the European Unions 7th FP (FP/2007-2013) / ERC GA n. 321253. It has been partially funded by the Spanish Government (TIN2014-34557).
- When compared to the baseline multi-core system implemented on the Xilinx FPGA, the AMMC multi-core system achieves $6.8 \mathrm{x}$ of speed-up, transfers datasets up to $1.95 \mathrm{x}$ faster, consumes $48 \%$ less hardware resources and $27.9 \%$ less on-chip power.

\section{AdVANCED Multi-CORE MEMORY CONTROLler}

In this section, we describe the AMMC system. The architecture (shown in Figure 1) is divided into five units: the Bus System (A), the Local Memory Unit (B), the Memory Manager (C), the Scheduler (D) and the Pattern Aware SDRAM Controller (E). The main units of AMMC are shown in Figure 1, as well as the Multi-Core System, that executes the applications. The Multi-Core System can have general purpose processors, application specific accelerator cores or a combination of both types. The Bus System [2] provides a link between AMMC and the Multi-Core System. The AMMC Memory Unit stores both data and the access pattern descriptors in Specialized Memory and Descriptor Memory respectively. Each processing core has a separate Specialized Memory [3] and a number of Descriptor Memory blocks. The descriptors are programmed at compile-time, providing information of the memory access patterns and their priorities. At run-time, the AMMC Scheduler receives multiple memory $\mathrm{read} /$ write requests from the Multi-Core System and selects a processing core, depending upon its priority level and scheduling policy. The Scheduler forwards the memory request to the Memory Manager. The Memory Manager is divided into the Address Manager and the Data Manager. The Address Manager takes a Task ID from the AMMC Scheduler and fetches its Descriptor Memory. Depending on the access pattern the Address Manager uses single or multiple descriptors, maps and rearranges addresses in hardware. The Address Manager saves mapped addresses into its Address Buffer for further reuse. The Data Manager improves the Computational Intensity [3] by organizing and managing the memory accesses. For a core processing a single computed point, the maximum achievable (ideal) Computational Intensity is 1 . The Data Manager accesses the elements in the form of patterns which are required for a single output (Computed Clement $_{\text {Cof }}$. After accessing the first access pattern, the Data Manager reuses and updates data where required. The Pattern Aware SDRAM Controller [3] is used to transfer data between main memory and the Specialized Memory.

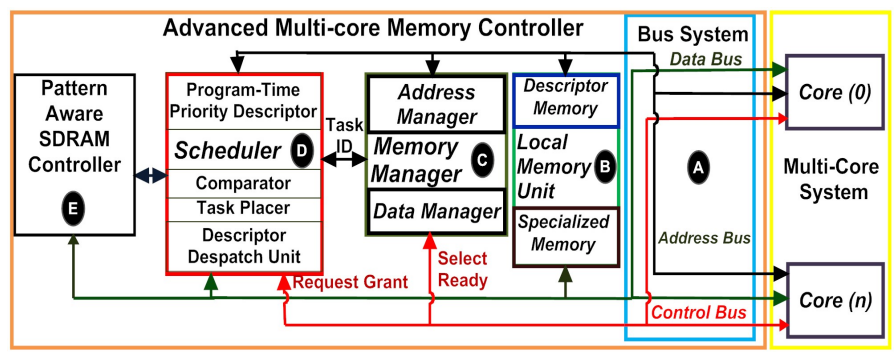

Fig. 1. Architecture of the Advanced Multi-core Memory Controller 


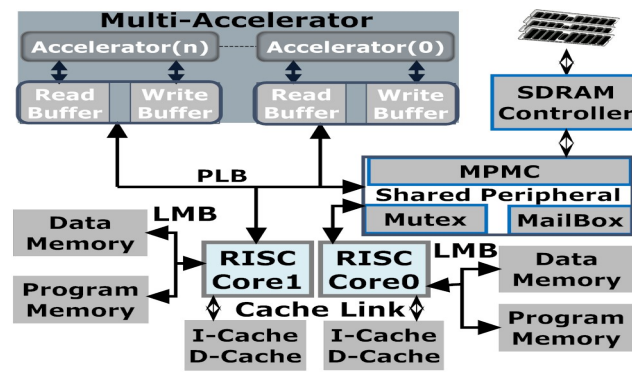

(a)

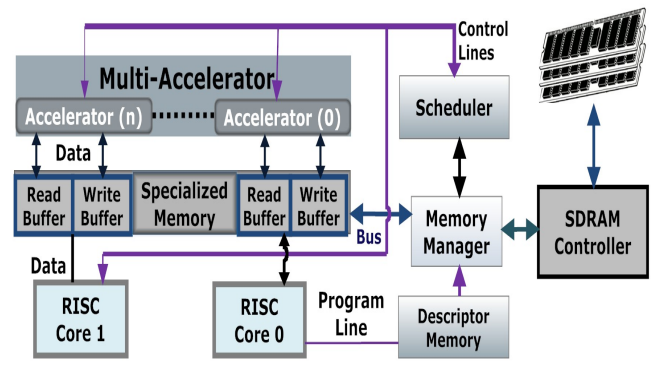

(b)

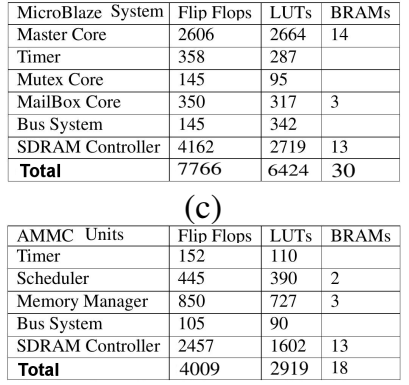

(d)

Fig. 2. Multi-Core Systems: (a) MicroBlaze (b) AMMC (c) MicroBlaze Resource Utilization (d) AMMC Resource Utilization

The AMMC Descriptor Memory holds memory access and scheduling information of applications running on the MultiCore System. Each processing core has a separate block of Descriptor Memory. The set of parameters for a Descriptor Memory block includes Command, Task ID, External Address, Priority, Size, Stride and Offset. Command specifies whether to read or write data. The Task ID and External Address parameters hold the address of processing core (buffer) and main memory (SDRAM) data set respectively. The Priority defines the order in which memory accesses are entitled to be process. The parameters Size and Stride define the type of memory access. The Offset register field is used to point the next linked memory access pattern.

The AMMC Scheduler manages and controls the run-time requests and programmed priorities of processing cores. Each processing core's request includes a read and write memory operation. At program-time, each processing core is assigned a priority value along with the Task $I D$, which are placed in the Program-Time Priority Descriptor (shown in Figure 1). The processing cores are categorized into three states, busy (core is processing on local buffer), requesting (core is idle), and request \& busy. In the request \& busy state the core is assumed to have double or multi buffers. During this state, the core is processing the data of one buffer while making a request to fill another buffer.

AMMC Scheduler supports two scheduling strategies of the requests of the cores: symmetric and asymmetric. The

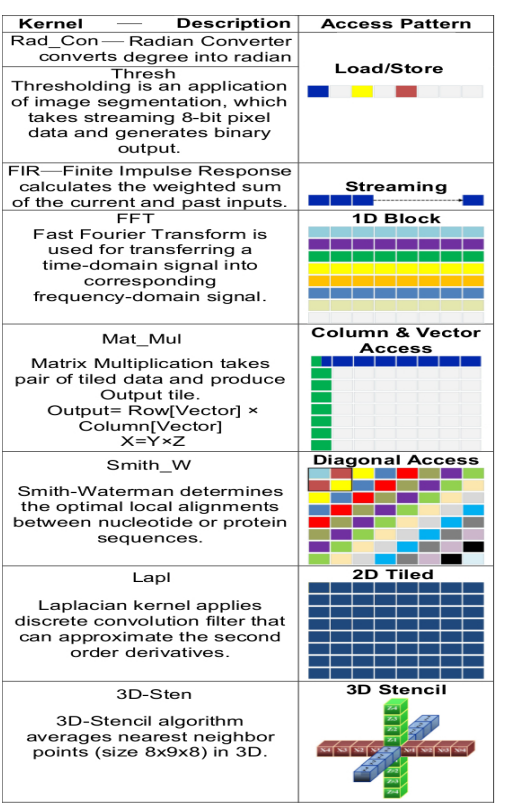

(a)

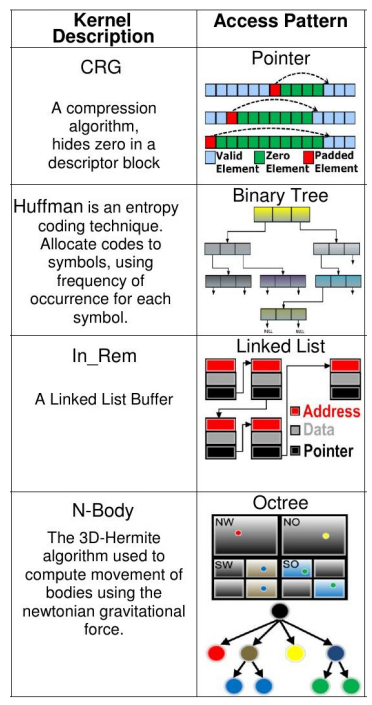

(b)
Fig. 3. Application Kernels: (a) Regular Access Pattern (b) Irregular Access Pattern scheduling policies are programmed statically at programtime and are executed by hardware at run-time. In Symmetric multi-core strategy, the AMMC Task Placer (Figure 1) manipulates the incoming requests in FIFO (First in First out) order and places them in the Dispatch Descriptor. The Asymmetric strategy uses the priority specified for each core and incoming requests. Each core is assigned a fixed priority at program-time, which is placed in Program-Time Priority Descriptor. At run-time, the Scheduler accumulates requests from the Multi-Core System. The Comparator and Task Placer maintain them in the Dispatch Descriptor. The Comparator takes requests from multiple processing cores, compares them with programmed priorities and forwards the results to Task Placer. The Task Placer places the requests in the Descriptor Dispatch Unit and executes requests only if it is ready to run, and there are no higher priority cores that are in ready state. The Dispatch Descriptor executes processing core requests sequently.

\section{EXPERIMENTAL FRAMEWORK}

In this section, we describe the MicroBlaze- and AMMCbased Multi-Core Systems and the rest of our experimental setup. A Xilinx ML505 evaluation FPGA board is used to test the Multi-Core Systems. The Xilinx Integrated Software Environment and Xilinx Platform Studio are used to design the Multi-Core Systems. Xilinx Power Estimator does the power analysis. The section is divided into three subsections: the Computation Units, the MicroBlaze based Multi-Core System and the AMMC based Multi-Core System.

\section{A. Computation Units}

There are two cores in our heterogeneous system: ASHA cores execute application kernels with regular memory accesses while SSP cores execute application kernels with irregular memory access patterns. Figure 3 lists all applications used in our experiments. The ASHAs are generated by the ROCCC [4] compiler. We have chosen the MicroBlaze SSP to implement the general purpose cores of our system. Microblaze is an RISC SSP architecture, optimized and implemented with FPGA resources. The multi-core system includes 2 MicroBlaze cores that run 2 applications each and 8 ASHA cores.

\section{B. MicroBlaze-based Multi-Core System}

The MicroBlaze-based Multi-Core System is used as baseline (Figure 2(a)). The resources utilized by the MicroBlaze based multi-core system is shown in Figure 2(c). Each general purpose core has $32 \mathrm{~KB}$ of data cache, that is implemented using BRAM. The design uses Xilinx Cache Links (IXCL/DXCL) for I-Cache and D-Cache memory accesses respectively. MicroBlaze instruction prefetcher improves the system performance by using the instruction prefetch buffer and instruction cache streams. 


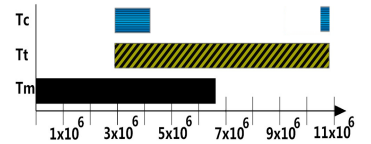

(a)

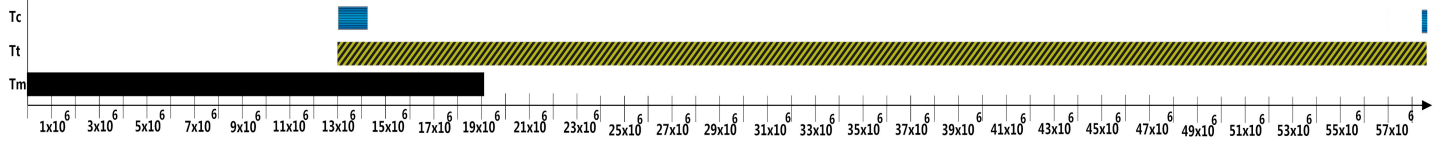

(b)

Fig. 4. Symmetric System Performance: (a) AMMC (b) MicroBlaze

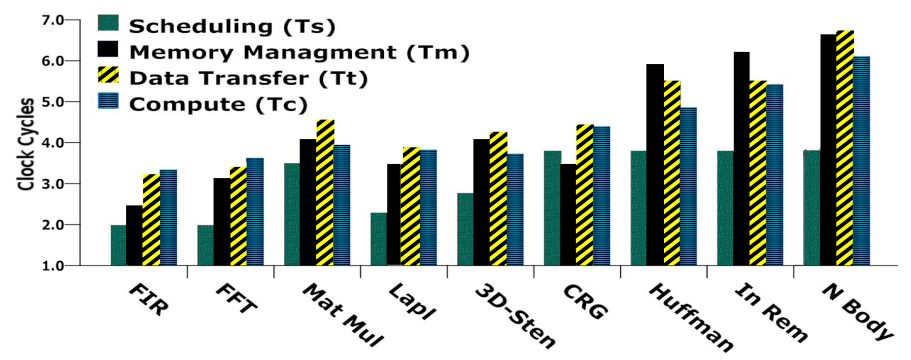

(a)

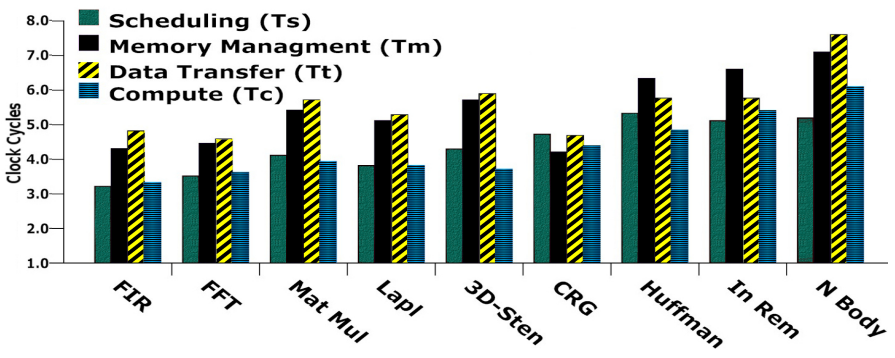

(b)

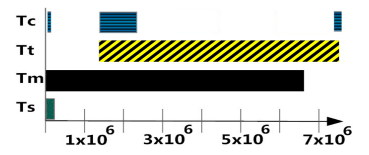

(c)

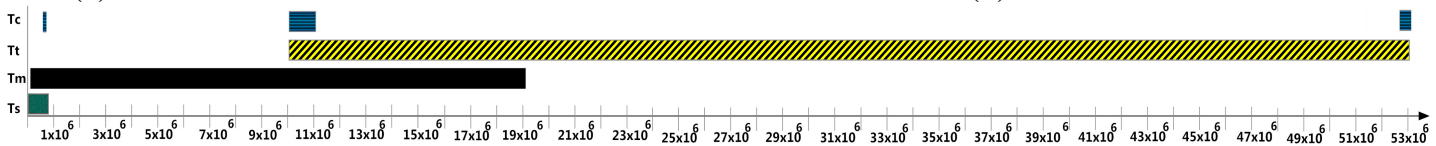

(d)

Fig. 5. Asymmetric System Performance: (a) AMMC (b) MicroBlaze (c) \& (d) AMMC \& MicroBlaze Systems Pipeline and Overlap Time Period

TABLE I. ASYMmetric SCHEdUling PRiority POLICIES

\begin{tabular}{|c|c|c|c|c|c|c|c|c|c|c|}
\hline Kernels & FIR & FFT & Mat_Mul & Lapl & 3D-Sten & CRG & Huffman & In_Rem & N-Body & Speed-ups \\
\hline Symmetric & I & 1 & 1 & 1 & 1 & 1 & 1 & 1 & 1 & $5.47 \mathrm{x}$ \\
\hline Asymmetric & 1 & 4 & 5 & 3 & 2 & & & & & \\
\hline $\begin{array}{l}\text { Group } 1 \\
\text { Group II }\end{array}$ & 2 & 3 & 4 & 5 & 1 & 8 & 6 & $\begin{array}{l}\circ \\
9\end{array}$ & 7 & $5.83 \mathrm{x}$ \\
\hline Group III & 9 & 6 & 5 & 4 & 8 & 4 & 3 & 2 & 1 & $3.45 \mathrm{x}$ \\
\hline Architecture & 1 & 1 & 1 & 1 & 1 & 2 & 2 & 2 & 2 & $5.42 \mathrm{x}$ \\
\hline
\end{tabular}

One of the MicroBlaze softcores (Core 0, Figure 2(a)) is the master core and is used to schedule the memory requests and to manage data transfer between multi-cores and main memory (SDRAM). The MicroBlaze cores use Xilkernel a small lightweight easy-to-use Real-Time Operating System (RTOS). Its API performs scheduling, inter-process communication and synchronization with POSIX threads (pthreads). From the main function, application spawns into multiple statically declared threads using the pthread library. Each thread controls a single application kernel and manages its memory patterns.

\section{AMMC based Multi-Core System}

Figure 2(b) shows the implementation of an AMMC-based Multi-Core System. SSP cores do not integrate any cache but use the local memory provided by AMMC. Similarly, there is no need for an RTOS like Xilkernel. The resources consumed by each AMMC unit is shown in Figure 2(d).

\section{RESULTS AND DisCUSSION}

This section analyzes the results of experiments conducted on AMMC and MicroBlaze based system. The experiments are characterized into two subsections: System Performance and Area \& Power.

\section{A. Multi-Core System Performance}

The system performance is measured by executing application kernels simultaneously using different scheduling policies, on AMMC and MicroBlaze based systems. Due to the confined FPGA resources, 5 ASHA and 2 SSP cores are integrated with the Multi-Core System. The execution time of both systems is categorized into four factors: scheduling time $(T s)$, memory management time $(T m)$, data transfer time $(T t)$ and computation time $(T c)$. Ts holds the arbitration (request, grant and wait) time among the on-chip scheduling. Tm comprises the address generation and data management time. $T t$ presents the data access time from external memory. It includes address mapping from physical address space to SDRAM address space, interface timing and synchronization. $T c$ holds the computation time of the application kernels. To measure the overlap and processing time, each application kernel is assigned four timers which count $T s, T m, T t$ and Tc clocks.

In the symmetric scheduling policy, the requests are treated with the FIFO method, which removes the scheduling time. Figures 4(a) and (b) present the overlapped/pipelined time of AMMC and MicroBlaze systems respectively. $\mathrm{X}$ and $\mathrm{Y}$ axis present clock cycles and execution time factors, respectively. While running the Multi-Core System using symmetric scheduling, the results show that the AMMC system achieves 5.47x of speed-up. The current Computation Units contain application kernels with different access patterns. The symmetric scheduling policy gives higher priority to application kernels with many memory requests. These requests add on-chip bus and memory access delays, therefore the AMMC system does not fully overlap $T m \& T t$. These delays can be decreased by executing Multi-Core System with asymmetric scheduling policy.

We categorize the asymmetric scheduling policy into two types; the memory access based asymmetric policy and the architecture based asymmetric policy (shown in Table I). The memory access based asymmetric policy assigns priorities to the application kernels with respect to their access patterns and is further categorized into three groups. In Group I, the highest priorities (1) are allocated to application kernels having less memory requests and dense access patterns. For example, the applications having multiple read/write requests are given low priorities. To check the sensitivity of asymmetric scheduling execution of the assigned priorities, the priorities of Group I are slightly varied in Group II. In Group III, the priorities 
are assigned to check the fairness of applications for priorities not assigned properly. For example, the highest priority (1) is allocated to application kernels having the largest amount of memory requests. Like MicroBlaze Xilkernel scheduling model, the AMMC scheduling policies and memory accesses are configured statically at program-time. Unlike Xilkernel, the requests are managed and executed by hardware at run-time. The memory access based asymmetric policy performs load balancing and reduces on-chip communication and memory management delay.

Figures 5(a) \& (b) present clock cycles of AMMC and MicroBlaze systems respectively, while executing application kernels simultaneously using memory access based asymmetric scheduling policy. $\mathrm{X}$ (logarithmic scale) and $\mathrm{Y}$ axis present clock cycles and application kernels, respectively. Each bar represents $T s, T m, T t$ and $T c$. While running all application kernel together using the asymmetric scheduling, the results show that the scheduling, memory manager and memory transfer of AMMC based system are $21 \mathrm{x}, 2.9 \mathrm{x}$ and 7.1x faster respectively, compared to the MicroBlaze based system. The computation units execution time $(T c)$ remains the same for both systems. Figures 5(c) and (d) present the overlapped/pipelined time of AMMC and MicroBlaze systems respectively. The $T c$ of all application kernels is overlapped (shown in Figure 5(c)). In the AMMC system, $T t$ and $T m$ are dominant for the regular and irregular application kernels respectively. As all AMMC units operate in parallel, AMMC overlaps all other units under the unit that consumes more time. For example for regular application kernels $T s, T m$ and $T c$ are overlapped under Tt. The MicroBlaze based system overlaps $T c \& T s$ completely and partially overlaps $T m$ and Tt (shown in Figure 5(d)). While running all application kernel together using the asymmetric scheduling with priorities of Group I, the results show that the AMMC based system achieves $6.84 \mathrm{x}$ of speed-up compared to the MicroBlaze based system. While executing application kernels with priories of Group II and Group III, the AMMC based system achieves 5.83 and $3.45 x$ of speed-up respectively. The AMMC asymmetric scheduling policy manages system resources (Application code, On-Chip Off-Chip Memory) of the Multi-Core System without the support of the operating system.

In the architecture based asymmetric policy, the Computation Units are assigned priorities depending upon their instruction set architecture, execution and communication (request/grant) speed. The architecture based asymmetric priorities are shown in Table I. All the cores of one type get the same priority. The priority 1 executes $A S H A$ core requests with higher priority. Requests having same priorities are executed in FIFO order. While running the Multi-Core System using the architecture based asymmetric scheduling policy, the results show that the AMMC based system achieves 5.42x of speedup compared to MicroBlaze based system. For performance evaluations, we analyzed that the priority based scheduling has the potential for supporting scalability and load balancing.

\section{B. Area \& Power}

Xilinx V5-Lx110T device dissipates 3.15 watts of on-chip static power, while running the MicroBlaze based system. The AMMC system draws 2.27 watts of on-chip power on a V5Lx110T device. While comparing the AMMC and MicroBlaze systems without slave units (accelerators and processor), results show that AMMC system consumes $48 \%$ fewer slices and $27.9 \%$ less on-chip static power than the MicroBlaze system. The AMMC provides low-power and simple control characteristics by rearranging data accesses and utilizing hardware units efficiently.

\section{RELATED WORK}

Marchand et al. [5] have developed software and hardware implementations of the Priority Ceiling Protocol that control the multiple-unit resources in a uniprocessor environment. Yan et al. [6] has designed a hardware scheduler to assist the synergistic processor cores (SPCs) task scheduling on heterogeneous multi-core architecture. The scheduler supports first come first service (FCFS) and dynamic priority scheduling strategies. It acts as helper engine for separate threads working on the active cores. The scouting hardware thread [7] tends to reduce latency, but also optimizes memory bandwidth usage by predicting memory accesses and by prioritizing valuable memory traffic using a separate core. The information of memory accesses is stored thus helping the scouting core to fetch and update data from the cache. The AMMC holds information of memory patterns in the form of Descriptor Memory. Currently, accessed patterns are placed in the address manager of AMMC. The AMMC monitors the access patterns without using a separate core and reuses these patterns for multiple cores if required.

Hussain et al. [8] [9] discussed a programmable pattern based memory controller architecture. The design is appropriate for data intensive applications with regular access patterns only. He also proposes a controller [3] that supports irregular applications running on a single core. Whereas in AMMC, we present a mechanism that supports both application-specific accelerators and RISC cores in a heterogeneous multi-core system having regular and irregular memory access patterns.

\section{CONCLUSION}

In this work, we have proposed AMMC that schedules multi-core operations while taking processing, scheduling, memory management and memory transfer into account. The AMMC architecture supports two types of cores: the general purpose RISC core and application specific hardware accelerator core. The AMMC improves the system performance by reducing the speed gap between accelerators/processors and memory and by scheduling/managing complex memory patterns without master core intervention. The AMMC system is implemented and tested on a Xilinx ML505 evaluation FPGA board. The performance of the system is compared with a microprocessor based system that has been integrated with the Xilkernel operating system. Results show that the AMMC based multi-core system consumes $48 \%$ fewer slices and $27.9 \%$ less on-chip static power and achieves $6.8 \mathrm{x}$ of speed-up compared to the MicroBlaze-based multi-core system having real time operating system.

\section{REFERENCES}

[1] András Vajda et al. Programming many-core chips. Springer, 2011.

[2] Tassadaq Hussain et al. MAPC: Memory Access Pattern based Controller. In 24th International Conference on FPL, 2014.

[3] Tassadaq Hussain et al. Advanced Pattern based Memory Controller for FPGA based Applications. In 24th International Conference on HPCS.

[4] Jason Villarreal et al. Designing modular hardware accelerators in c with roccc 2.0. In FCCM 2010.

[5] P. Marchand and P. Sinha. A hardware accelerator for controlling access to multiple-unit resources in safety/time-critical systems. Inderscience Publishers, April 2007.

[6] L. Yan et al. Hardware Assistant Scheduling for Synergistic Core Tasks on Embedded Heterogeneous Multi-core System. In Journal of Information \& Computational Science (2008).

[7] Shailender et al. Chaudhry. Simultaneous speculative threading: a novel pipeline architecture implemented in sun's rock processor. ACM, 2009.

[8] T. Hussain, M. Shafiq, M. Pericas, N. Nacho and E. Ayguade. PPMC: A Programmable Pattern based Memory Controller. In ARC 2012.

[9] Tassadaq Hussain and Amna Haider. PGC: A Pattern-Based Graphics Controller. Int. J. Circuits and Architecture Design, 2014. 